\title{
Leptomeningeal Metastases of the Spine: A Systematic Review
}

\author{
PAOLO PALMISCIANO ${ }^{1}$, NAVRAJ S. SAGOO ${ }^{2}$, ABDURRAHMAN F. KHARBAT $^{3}$, \\ YVES J. KENFACK ${ }^{4}$, OTHMAN BIN ALAMER ${ }^{5}$, GIANLUCA SCALIA ${ }^{6}$, \\ GIUSEPPE E. UMANA ${ }^{1}$, SALAH G. AOUN ${ }^{7}$ and ALI S. HAIDER ${ }^{8,9}$ \\ ${ }^{1}$ Department of Neurosurgery, Trauma Center, Gamma Knife Center, Cannizzaro Hospital, Catania, Italy; \\ ${ }^{2}$ Department of Orthopaedic Surgery, University of Texas Southwestern Medical Center, Dallas, TX, U.S.A.; \\ ${ }^{3}$ Texas Tech University Health Sciences Center School of Medicine, Lubbock, TX, U.S.A.; \\ ${ }^{4}$ University of Texas Southwestern Medical Center, Dallas, TX, U.S.A.; \\ ${ }^{5}$ King Abdullah International Medical Research Center, Riyadh, Kingdom of Saudi Arabia; \\ ${ }^{6}$ Department of Neurosurgery, Highly Specialized Hospital of National Importance "Garibaldi", Catania, Italy; \\ ${ }^{7}$ Department of Neurological Surgery, University of Texas Southwestern Medical Center, Dallas, TX, U.S.A.; \\ ${ }^{8}$ Texas A\&M University College of Medicine, Houston, TX, U.S.A.; \\ ${ }^{9}$ Department of Neurosurgery, The University of Texas MD Anderson Cancer Center, Houston, TX, U.S.A.
}

\begin{abstract}
Background/Aim: Leptomeningeal metastases (LMs) of the spine have complex management. We reviewed the literature on spine LMs. Materials and Methods: PubMed, EMBASE, Scopus, Web-of-Science, and Cochrane were searched following the PRISMA guidelines to include studies of spine LMs. Results: We included 46 studies comprising 72 patients. The most frequent primary tumors were lung (19.4\%) and breast cancers $(19.4 \%)$. Median time from primary tumors was 12 months (range=0-252 months). Cauda equina syndrome occurred in 34 patients (48.6\%). Nodular spine LMs (63.6\%) were more frequent. Concurrent intracranial LMs were present in 27 cases (50.9\%). Cerebrospinal fluid cytology was positive in 31 cases (63.6\%). Cases were managed using palliative steroids $(73.6 \%)$ with locoregional radiotherapy (55.6\%) chemotherapy (47.2\%), or decompressive laminectomy (8.3\%). Post-treatment symptom improvement (32\%) and favorable radiological response $(28.3 \%)$ were not different based on treatment $(p=0.966 ; p=0.727)$. Median overallsurvival was 3 months (range $=0.3-60$ months), not significantly
\end{abstract}

This article is freely accessible online.

Correspondence to: Paolo Palmisciano, MD, Department of Neurosurgery, Trauma Center, Gamma Knife Center, Cannizzaro Hospital, Via Messina 829, 95126 Catania, Italy. Tel: +39 3331751077, e-mail: paolo.palmisciano94@gmail.com

Key Words: Leptomeningeal metastases, radiotherapy, spine metastases, spine surgery, spine tumors, systematic review. different between radiotherapy and chemotherapy $(p=0.217)$. Conclusion: Spine LMs have poor prognoses. Radiotherapy, chemotherapy, and surgery are only palliative, as described for intracranial LMs.

Leptomeningeal metastases (LMs) are late-stage complications of systemic malignancies, occurring approximately in 5-10\% of patients with solid and hematologic neoplasms $(1,2)$. Frequently manifesting with new neurological deficits, LMs may be detected at T1-contrast MRI follow-ups as nodular or diffuse meningeal enhancement, and may be confirmed with high-volume cerebrospinal fluid (CSF) taps for cytology (3, 4). Several systemic therapeutic options are available, but the treatment goal remains palliation, as mean survival ranges 36 months $(3,5,6)$.

LMs involving the spine represent unique entities that may severely impact patients' functional status $(7,8)$. While diffuse spinal LMs may remain clinically silent and go undetected, nodular spinal LMs frequently compress spinal nerve roots causing radicular pain or neurological impairments $(2,9)$. Nodular spinal LMs may also lead to acute or progressive cauda equina syndrome, which require urgent diagnosis and management $(10,11)$. While surgical decompression may provide prompt symptomatic relief, patients may not be good candidates due to their significant tumor burden $(2,3)$. In these cases, locoregional radiation, chemotherapy, and other systemic treatments may be pursued to achieve similar clinical outcomes $(12,13)$.

Although spinal LMs pose significant challenges in the oncological care of patients with systemic metastases, only 
few case reports and small-sized case series have been reported, and no standardized management workups have been defined. In this study, we systematically reviewed the literature to summarize the clinical characteristics of patients with spinal LMs, analyzing the impact of available therapeutic options on patients' quality-of-life and survival.

\section{Materials and Methods}

Literature search. A systematic review was conducted according to the Preferred Reporting Items for Systematic Reviews and MetaAnalyses (PRISMA) guidelines (14). PubMed, EMBASE, Scopus, Web of Science and Cochrane were searched from database inception to August 27, 2021 operating the Boolean full-text search [(leptomeningeal disease OR leptomeningeal metastases OR leptomeningeal carcinomatosis) AND (spine OR vertebral OR sacral)]. Studies were exported to Mendeley, and duplicates deleted.

Study selection. Inclusion and exclusion criteria were defined. Articles were included if they: 1) involved $\geq 1$ patients with radiological diagnosis of LMs involving the spine and originating from primary non-central nervous system (CNS) tumors; 2) reported clinical, treatment, and outcomes data; 3) were written in English. Studies were excluded if they: 1) were reviews, autopsy reports, or animal studies; 2) involved patients with spinal LMs from primary CNS tumors; 3) did not differentiate data of patients with spine LMs from patients only with intracranial LMs; 4) lacked adequate data on treatment outcomes.

Two independent reviewers (A.F.K. and P.P.) screened all titles and abstracts and then assessed full-texts of articles that met the inclusion criteria. Disagreements were settled by a third reviewer (N.S.S.). Eligible papers were included, and references were screened to identify additional pertinent studies.

Data extraction. One reviewer (Y.J.K.) extracted data from each article, then confirmed independently by two additional reviewers (P.P. and A.S.H.). Extracted data included: author, study design, sample size, age, gender, primary tumor, preceding metastases and treatments, time interval between primary tumor and spine LMs, spine LMs' presenting symptoms and location, concurrent intracranial LMs, spine LMs radiological appearance, CSF cytology, treatment strategies (including surgery, steroids, radiotherapy, chemotherapy, or additional therapies), clinical response, spine LMs radiological response, spine LMs recurrence, local control (LC), overall survival (OS), survival status. Clinical and radiological responses were measured at last available follow-up. Clinical responses were evaluated based on post-treatment patients' functional status compared to pre-treatment status as reported by the authors, divided into: resolution of baseline symptoms, improvement of baseline symptoms, no change in baseline symptoms (stable), worsening of baseline symptoms. Radiological responses were assessed based on the RANO criteria modified for LMs, describing changes in pathological spine T1-contrast enhancement from baseline: complete response (CR) - no evidence of disease -, partial response (PR) - decrease by $>50 \%$ in the summed product of orthogonal diameters of composite measurable nodules -, stable disease (SD) - no change of disease -, progressive disease (PD) increased by $>25 \%$ in the summed product of orthogonal diameters of composite measurable nodules or new sites of disease (15).
Data synthesis and quality assessment. Primary variables of interest were clinical characteristics, management strategies, and treatment outcomes of patients with spine LMs. For each study, two independent authors (P.P. and N.S.S.) assessed level of evidence using the 2011 Oxford Centre For Evidence-Based Medicine guidelines, and risk of bias applying the Joanna Briggs Institute checklists for case reports and case series (16-18). Meta-analysis was precluded because all included studies had levels IV-V of evidence, and hazard ratios could not be deducted.

Statistical analysis. SPSS V.25 (IBM Corp, Armonk, NY, USA) was used for all statistical analyses and two-sided $p$-values $<0.05$ were considered significant. Continuous variables are presented as medians and ranges, and categorical variables as frequencies and percentages. Chi-square test was used to analyze differences in post-treatment symptom improvement between patients receiving locoregional spine radiotherapy and patients receiving chemotherapy. Time intervals between last treatment and spine LMs recurrence (LC curve) or death (OS curve) were estimated using the Kaplan-Meier method, and the survival analyses were conducted using the log-rank test.

\section{Results}

Study selection and overview. The search strategy yielded 2727 studies (PubMed: 1378, EMBASE: 1083, Web of Science: 207, Scopus: 54, Cochrane: 5), of which 46 were included in the qualitative synthesis (Supplementary File 1). Five were case series (including 31 patients) and 41 were case reports, with IV and V levels of evidence, respectively (Supplementary File 2) (13, 19-64). Critical appraisal returned low risk of bias for all included studies (Supplementary File 3).

Demographics and primary tumors' characteristics. A total of 72 patients diagnosed with spine LMs were analyzed (Table I). The median age at diagnosis was 51 years (range $=29-87$ years), with male predominance $(52.9 \%)$. The most common primary tumors comprised breast cancer $(19.4 \%)$, non-small cell lung carcinoma (19.4\%), and lymphoma (9.7\%). One patient $(1.6 \%)$ was diagnosed with a metastatic carcinoma of unknown primary (CUP) (45). Secondary systemic metastases preceding the onset of spine LMs were detected in 39 patients $(54.9 \%)$, most frequently occurring in the brain parenchyma $(38.9 \%)$ and/or regional lymph nodes $(18.1 \%)$. Metastatic peritoneal seeding was described in 4 patients with gynecological neoplasms (22, $50,51,63)$ and 1 patient with gastric adenocarcinoma (27). While primary tumors were treated with variable cancer-specific multidisciplinary strategies including surgery, adjuvant chemotherapy, and radiation, brain metastases were preferentially treated with conventional or stereotactic radiotherapy $(66.7 \%)$ and/or surgical resection when feasible $(60 \%)$.

Clinical and diagnostic features of spine LMs. The median time interval between primary tumors and spine LMs was 12.0 months (range $=0-252.0$ months), with spine LMs 
Table I. Summary of patients' demographics and primary tumors characteristics.

\begin{tabular}{|c|c|}
\hline $\begin{array}{l}\text { Characteristics } \\
\text { (no. of patients for whom } \\
\text { information is available) }\end{array}$ & $\begin{array}{c}\text { Value } \\
\text { (among patients } \\
\text { with available data) }\end{array}$ \\
\hline Cohort size (no.) & 72 \\
\hline \multicolumn{2}{|l|}{ Demographics $(n=70)$} \\
\hline Median age, range (years) & $51,29-87$ \\
\hline Gender (male) & $37(52.9 \%)$ \\
\hline Primary Tumor $(n=72)$ & No. $(\%)$ \\
\hline Breast & $14(19.4 \%)$ \\
\hline Lung (non-small cell carcinoma) & $14(19.4 \%)$ \\
\hline Lymphoma & $7(9.7 \%)$ \\
\hline Esophageal & $5(6.9 \%)$ \\
\hline Chronic lymphocytic leukemia & $3(4.2 \%)$ \\
\hline Colorectal & $3(4.2 \%)$ \\
\hline Nasopharyngeal & $3(4.2 \%)$ \\
\hline Ovarian & $3(4.2 \%)$ \\
\hline Prostatic & $3(4.2 \%)$ \\
\hline Renal & $3(4.2 \%)$ \\
\hline Cervix & $2(2.8 \%)$ \\
\hline Gastric & $2(2.8 \%)$ \\
\hline Melanoma & $2(2.8 \%)$ \\
\hline Pancreas & $2(2.8 \%)$ \\
\hline Skin - Squamous cell carcinoma & $2(2.8 \%)$ \\
\hline Bladder & $1(1.4 \%)$ \\
\hline Carcinoma of unknown origin & $1(1.4 \%)$ \\
\hline Plasmocytoma & $1(1.4 \%)$ \\
\hline Testicular & $1(1.6 \%)$ \\
\hline Secondary metastases $(n=72)$ & No. $(\%)$ \\
\hline $\begin{array}{l}\text { Patients with systemic metastases } \\
\text { preceding spine LMs }\end{array}$ & $39(54.9 \%)$ \\
\hline Brain & $28(38.9 \%)$ \\
\hline Lymph nodes & $13(18.1 \%)$ \\
\hline Lung & $7(9.7 \%)$ \\
\hline Bone (Long bones, Spine, Skull base) & $5(6.9 \%)$ \\
\hline Peritoneal seeding & $5(6.9 \%)$ \\
\hline Liver & $4(5.6 \%)$ \\
\hline Treatment brain metastases $(n=15)$ & No. $(\%)$ \\
\hline Radiation Therapy & $10(66.7 \%)$ \\
\hline Surgery & $9(60 \%)$ \\
\hline $\begin{array}{l}\text { Time between primary tumor and } \\
\text { spine LMs, months (range) }\end{array}$ & $12.0(0-252.0)$ \\
\hline
\end{tabular}

LM: Leptomeningeal metastases.

preceding primary tumor diagnosis in 4 cases $(43,45,47$, $60)$. The most common presenting symptoms were sensory $(50 \%)$ and motor $(45.7 \%)$ deficits, with cauda equina syndrome documented in 34 patients $(48.6 \%)$ (Table II). Dagan et al. (53) reported one case of cervical LM detected at follow-up imaging in one patient with no new spinerelated symptoms. Spine LMs were most frequently diagnosed at MRI T1-contrast scans with or without CSF cytologic analysis ( $66.7 \%$ and $25 \%$ cases, respectively), and confirmatory histopathological diagnosis was obtained only in 6 patients $(7.3 \%)(23,26,30,56,62)$. At neuroimaging,
Table II. Summary of clinical and diagnostic characteristics of spine leptomeningeal metastases.

\begin{tabular}{lc}
\hline $\begin{array}{l}\text { Characteristics } \\
\text { (no. of patients for whom }\end{array}$ & $\begin{array}{c}\text { Value } \\
\text { (among patients } \\
\text { available data) }\end{array}$ \\
information is available) & No. $(\%)$ \\
\hline Spine LMs presenting symptoms (n=70) & $35(50 \%)$ \\
Sensory Deficits & $34(48.6 \%)$ \\
Cauda equina syndrome & $32(45.7 \%)$ \\
Motor deficits & $26(37.1 \%)$ \\
Radicular pain & $24(34.3 \%)$ \\
Lower back pain & $16(22.9 \%)$ \\
Paraparesis & $7(10 \%)$ \\
Urine incontinence & $6(8.7 \%)$ \\
Ataxia & $3(4.3 \%)$ \\
Dorsal pain & $1(1.4 \%)$ \\
Cervical pain & $1(1.4 \%)$ \\
No symptoms & No. $(\%)$ \\
Diagnostic protocols (n=72) & $48(66.7 \%)$ \\
CSF cytology + Imaging & $18(25 \%)$ \\
Imaging only & $5(6.9 \%)$ \\
Imaging + Pathology & $1(1.4 \%)$ \\
CSF cytology + Imaging + Pathology & No. $(\%)$ \\
LMs location (n=72) & $16(22.2 \%)$ \\
Lumbar-Sacral & $13(18.1 \%)$ \\
Thoracic-Lumbar-Sacral & $9(12.5 \%)$ \\
Lumbar & $8(11.1 \%)$ \\
Cervical-Thoracic & $8(11.1 \%)$ \\
Thoracic-Lumbar & $6(8.3 \%)$ \\
Cervical-Thoracic-Lumbar-Sacral & $5(6.9 \%)$ \\
Thoracic & $4(5.6 \%)$ \\
Cervical & $3(4.2 \%)$ \\
Cervical-Thoracic-Lumbar & No. $(\%)$ \\
Spine leptomeningeal T1-Contrast & \\
enhancement (n=44) & $28(63.6 \%)$ \\
Nodular & $16(36.4 \%)$ \\
Diffuse & $27(50.9 \%)$ \\
Concurrent intracranial LMs (n=53) & No. $(\%)$ \\
Positive & $31(63.6 \%)$ \\
Negative & $18(36.4 \%)$ \\
\hline
\end{tabular}

CSF: Cerebrospinal fluid; LM: leptomeningeal metastases.

most spine LMs were located in the lumbar-sacral (18.1\%), thoracic-lumbar-sacral $(18.1 \%)$, or lumbar $(12.5 \%)$ regions, showing nodular $(63.6 \%)$ or diffuse $(36.4 \%)$ leptomeningeal enhancement. In 27 cases $(50.9 \%)$, concurrent intracranial LMs were described. Lumbar puncture and CSF cytology were performed in 49 patients $(69 \%)$, positive for tumor cells in 31 cases $(63.6 \%)$ and negative in $18(36.4 \%)$.

Management strategies. Palliative steroids were administered in 53 cases $(73.6 \%)$ for managing tumor-related edema and reduce spinal cord/nerve root compression (Table III). 
Table III. Summary of treatment strategies and outcomes of all pooled patients.

\begin{tabular}{lc}
\hline $\begin{array}{l}\text { Characteristics } \\
\text { (no. of patients for whom } \\
\text { information is available) }\end{array}$ & $\begin{array}{c}\text { Value } \\
\text { (among patients } \\
\text { with available data) }\end{array}$ \\
\hline Management strategies (n=72) & No. (\%) \\
Corticosteroids & $53(73.6 \%)$ \\
Spine radiation therapy & $40(55.6 \%)$ \\
Systemic chemotherapy & $34(47.2 \%)$ \\
Intrathecal methotrexate & $17(23.6 \%)$ \\
Decompressive laminectomy + LMs Removal & $6(8.3 \%)$ \\
Hormonal therapy & $2(2.8 \%)$ \\
Immunotherapy & $1(1.4 \%)$ \\
Post-treatment symptom improvement (n=53) & $17(32 \%)$ \\
& \\
Radiological response, RANO criteria, & No. $(\%)$ \\
last follow-up (n=53) & \\
Complete response (CR) & $5(9.4 \%)$ \\
Partial response (PR) & $10(18.9 \%)$ \\
Stable disease (SD) & $14(26.4 \%)$ \\
Progression (PD) & $24(45.3 \%)$ \\
Recurrence of spine LMs (n=72) & $4(5.6 \%)$ \\
Outcome (months) & \\
Median (range) & \\
Local control (n=72) & $2.0(0.3-60.0)$ \\
Overall survival (n=72) & $3.0(0.3-60.0)$ \\
Status (n=72) & No. $(\%)$ \\
Alive & $25(35.7 \%)$ \\
Dead & $47(65.3 \%)$ \\
\hline
\end{tabular}

LM: Leptomeningeal metastases; RANO: response assessment in neurooncology.

Locoregional radiotherapy was delivered in 40 patients (55.6\%), targeting the whole spine axis in 8 cases (20\%) and extending to the brain in $5(12.5 \%)$. Chemotherapy was administered in 34 patients (47.2\%), mostly with intrathecal methotrexate $(17 / 34,50 \%)$ and less frequently with variable cancer-specific protocols including fludarabine, ifosfamide, carboplatin, and etoposide. Decompressive laminectomy and surgical removal of nodular LMs were performed in 6 patients $(8.3 \%)$ with acute debilitating motor deficits and/or cauda equina syndrome, with no notable post-operative complications $(23,26,30,56,62)$. Concurrent hormonal therapies were administered in 2 patients $(2.8 \%)$ respectively for the treatment of spine LMs from prostate (leuprolide acetate)(34) and breast (anastrozole)(48) primary neoplasms, and immunotherapy with nivolumab (anti-PD1 monoclonal antibody; Bristol Myers Squibb, NY, NY, USA) for treating one patient with melanoma spine LM (57).

Treatment outcomes and survival. A total of 17 patients experienced post-treatment symptom improvement, with no differences based on locoregional spine radiotherapy vs. chemotherapy ( $p=0.245)$ (Table III). Radiological responses were evaluated in 53 patients in accordance with the RANO criteria modified for LMs, returning: CR in five (9.4\%), PR in 10 (18.9\%), SD in $14(26.4 \%)$ and PD in 24 (45.3\%) (15). Recurrences of spine LMs were documented in 4 patients (5.6\%) with median LC of 2.0 months (range=0.3-60.0 months). At last follow-up, most patients were dead (65.3\%) showing a median OS of 3.0 months (range=0.3-60.0 months), not significantly different between locoregional spine radiotherapy and chemotherapy $(p=0.217)$ (Figure 1$)$.

\section{Discussion}

LMs are debilitating complications of advanced systemic malignancies that rarely occur within the spine. Although progress in imaging and treatment protocols contributed to increased survival in oncological patients and higher incidence of spine LMs, optimal management remains uncertain $(3,4,6)$. We found that spine LMs severely worsen functional status of affected patients, leading to prompt treatment planning mostly for palliation. Still, currently available treatments proved to be limited in improving patients' quality-of-life and prognoses. This review aimed to offer valuable insights for the management of spine LMs, advocating the need to better understand such entities and devise appropriate therapies.

In this review of 72 patients, the most frequent primary tumors were breast cancer and non-small cell lung carcinoma, in line with their overall prevalence in the general oncological population (65). Our lower rate of primary hematologic malignancies reflects similar findings described in patients with intracranial LMs, likely implying different routes of tumor cell leptomeningeal spreading as compared to solid tumors $(2,3,12)$. Seeding of leptomeningeal tumors has been theorized to occur through several means, comprising: 1) hematogenous spread to the CSF, especially through the Batson plexus from pelvic tumors to spine LMs; 2) direct invasion from adjacent primary or metastatic tumors involving the dura or brain parenchyma, leading to intracranial LMs; 3 ) progression and extension through perineural and perivascular spaces, which may explain some cases of spine LMs $(66,67)$. The presence of systemic metastases and the surgical or radiation management of parenchymal brain metastases have been also suggested to facilitate intracranial and spine LMs, further confirmed by the high prevalence of preceding systemic (54.9\%) and brain (38.9\%) metastases in our cohort (65). While piecemeal resection and/or targeted radiation of brain metastases may cause the disruption of the blood-brain barrier (BBB) favoring direct trans-meningeal and intrasubarachnoid space spillage of cancers cells, current oncological therapies improve systemic disease control but poorly penetrate the $\mathrm{BBB}$, leading to tumor cell reservoirs within the CNS and higher risk of CSF spreading (68). For 

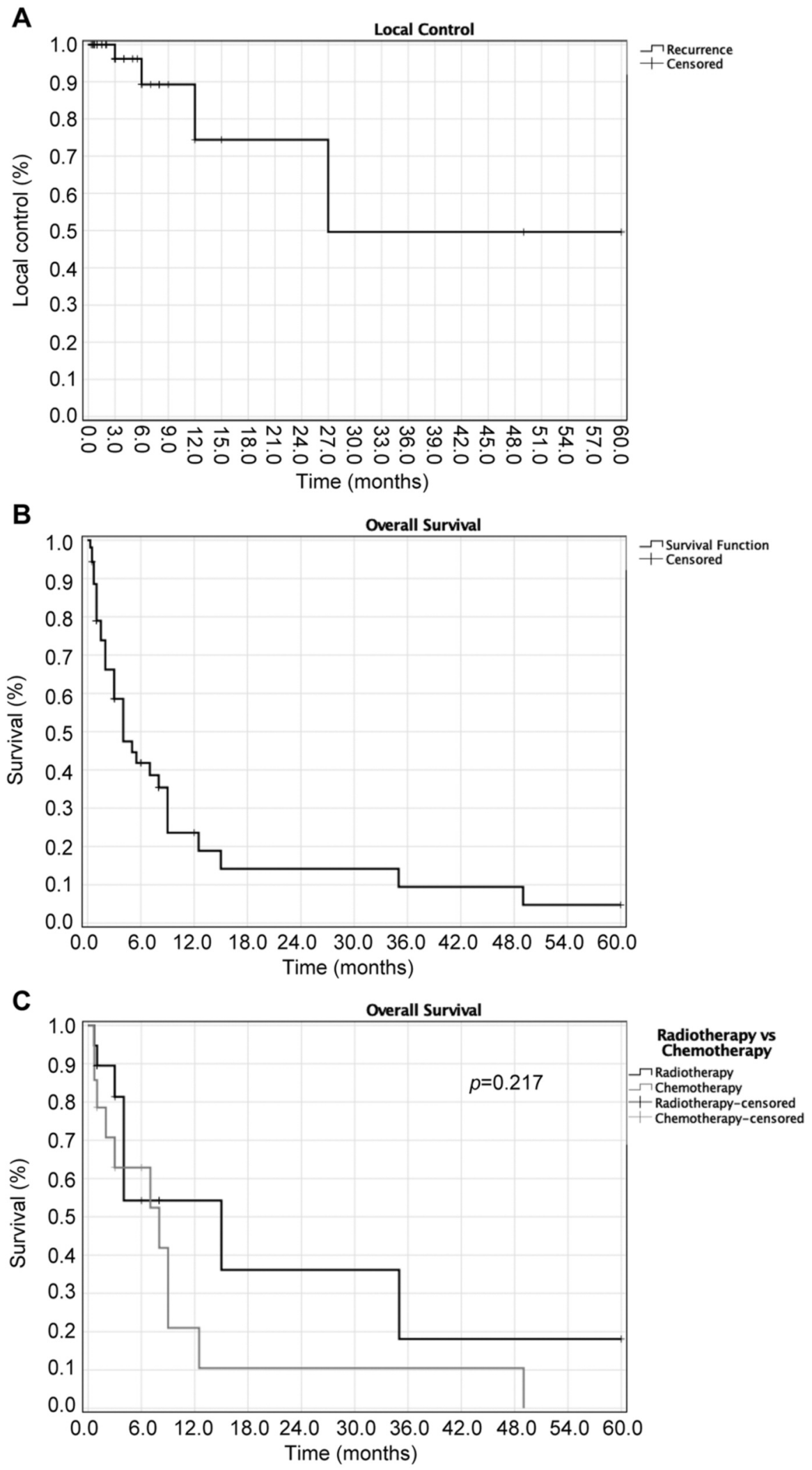

Figure 1. Kaplan-Meier survival curves of: (A) local control and (B) overall survival of the total pooled cohort; (C) overall survival based on locoregional radiotherapy vs. chemotherapy. 
these reasons, LMs from $\mathrm{CNS}$ and non-CNS tumors are frequently diagnosed at later disease stages, as we found in our pooled spine LMs cases, presenting with a median time interval of 12 months from primary tumor diagnosis $(3,8,69)$. However, as spine LMs may constitute the initial diagnosis of neoplastic diseases in few patients with slow-growing tumors, different leptomeningeal seeding mechanisms should be also considered and identified to guide timely diagnostic and therapeutic strategies $(43,45,47,60)$.

Clinical evaluation may support the initial diagnosis of secondary metastases involving the spine, but symptoms of LMs are mostly non-specific $(65,70)$. Similarly to spine LMs from primary CNS tumors, spine LMs from non-CNS tumors frequently present with sensorimotor deficits, lumbago, and/or radicular pain deriving from spinal cord and nerve root compression $(8,13,58)$. However, as these symptoms may also occur in other benign or malignant conditions, including intervertebral disc herniation or vertebral fracture, patients' history of oncological disease and systemic metastases should be investigated to plan appropriate metastatic diagnostic protocols $(71,72)$. The occurrence of cauda equina syndrome has also been largely reported across our included studies $(51,54-56)$. This likely owes to the fact that CSF circulation is slower in the cauda equina and the basilar cisterns, which represent the two regions mostly affected by leptomeningeal seeding $(66,67)$. Finally, the concurrent presence of intracranial LMs may also lead to a wide range of additional poorly-localized symptoms, such as headache and/or cranial nerve palsies, following CSF obstruction and increase in intracranial pressure $(28,30,61)$. Hence, the complex clinical presentation of patients with suspected secondary spine LMs highlights the importance to obtain MRI T1-contrasted scans and CSF cytology to assist the diagnostic workflow (15).

Brain and spine imaging represents the first diagnostic approach in patients with metastatic disease after the onset of new neurological impairments $(3,70)$. Early studies reported the use of diagnostic CT and/or myelogram exams, which identified suspected spine LMs as alterations of the spinal CSF circulation $(19,30,41)$. Contrast-enhanced MRI scans represent the current diagnostic gold-standard for detecting and localizing suspected LMs within the brain-spine axis $(6,11)$. We found that most spine LMs presented a nodular contrast-enhancing appearance and involved the lumbar-sacral or thoracic-lumbarsacral regions with cauda equina infiltration, explaining the corresponding clinical presentation patterns. As concurrent diffuse multifocal intracranial LMs have been documented in more than half of our pooled cases, we emphasize the importance to include brain axis imaging in known metastatic patients with suspected spine lesions or in patients with newly diagnosed spine LMs $(11,65)$. CSF cytologic analysis may be used to confirm the diagnosis of LMs by detecting pathological cellular lines similar to the primary tumors' ones $(38,46,54$,
57, 59). However, lumbar taps may be difficult to obtain, especially in patients with CSF obstruction, and negative CSF cytology may occur in up to $40 \%$ of patients with clinical and radiological diagnosis of $\operatorname{LMs}(6,65,69,73)$. Indeed, we found that $31.9 \%$ of our pooled patients were deemed not eligible to undergo lumbar tap due to poor functional status, and, among those with available CSF cytology, 36.4\% had negative results. Of note, 5 patients with initial negative CSF had positive results after a second lumbar tap and cytologic assessment, advocating the need to repeat CSF analysis in cases of dubious negative results $(13,39,43,65)$. Although blood biomarkers and liquid biopsy are gaining ground in LMs' diagnosis due to their minimal invasiveness and promising diagnostic accuracies, we found no reports among our included studies, which should raise interest in better evaluating such techniques for patients with spine LMs $(65,74)$.

Challenges in the management of spine LMs result from the poor clinical conditions of patients with systemic malignancies, coupled with the need to provide symptom relief and improve quality-of-life. Available therapies are mostly palliative, intended to ease metastatic tumor burden while avoiding treatment-related adverse events (75). For these reasons, corticosteroids were largely used in our pooled patients, mostly to provide alleviation of musculoskeletal and radicular pain. However, as the prolonged use of steroids may cause severe systemic complications with major impact on patients' functional status, several multimodal treatments have been described in spine LMs $(13,76)$. Locoregional spine radiotherapy has been delivered in most symptomatic patients with spine abnormalities at imaging. Similarly to intracranial cases, radiotherapy for spine LMs has the ability to reduce lesions' volumes and facilitate the CSF flow, relieving compression-related symptoms and improving the distribution of intrathecal agents when needed (12). In some patients with diffuse and multifocal LMs, radiation protocols targeting the whole spine axis or extending to the brain axis have been implemented for prolonging local tumor control $(28,46,49,55)$. These protocols should be carefully discussed in multidisciplinary teams, weighting the expected clinical benefits in such patients with dismal prognoses to the high risks of severe radiation-induced complications. The role of chemotherapy, with or without intrathecal administration, has also been largely studied in patients with LMs from CNS and non-CNS tumors, and various agents used also for spine LMs, as we found in this review $(8,75)$. In the current clinical practice, chemotherapeutic regimens are planned on the basis of primary tumors and anticipated responses. While systemic chemotherapy may be limited by the $\mathrm{BBB}$, showing better penetration in patients with $\mathrm{BBB}$ disruption, pharmacotherapy can be also delivered intrathecally via lumbar punctures or intraventricular devices to achieve superior concentrations within the CSF. Yet, the higher toxicity of intrathecal agents should be considered, 
especially in patients with LM-induced CSF obstruction (65, 75). As reported for patients with primary gliomas, surgery is limited for spine LMs, mostly intended to relieve compression-related symptoms with decompressive laminectomy and tumor debulking, but carries severe risks of complications and shows no impact on patients' prognoses $(8,23,26,56)$. Finally, newer molecular therapies and immunotherapies have been showing promising results for the treatment of intracranial LMs by simultaneously modulating microenvironments of primary tumors and metastatic lesions, but their role in spine LMs has been poorly studied $(69,77)$. In this review, Wu et al. (57) reported the unique case of melanoma nodular LM treated with lumbosacral radiotherapy followed by 11 cycles of nivolumab therapy. Prolonged local tumor control (27 months) and survival (35 months) were achieved, corroborating the theory that such strategies may serve as game-changers for the management of these complex patients and should be investigated on spine LMs.

In line with the current literature on intracranial LMs and spine LMs from primary CNS tumors, we found that patients with spine LMs from primary non-CNS tumors have poor prognoses, with median LC of 2 months and median OS of 3 months (range $=0.3-60$ months) $(8,65)$. This may be explained by the fact that spine LMs occur at later disease stages in patients with multiple concurrent systemic metastases and high tumor burden, which vastly limit the survival benefit of available treatments, as confirmed by the absence of survival differences between radiotherapy and chemotherapy in our cohort. For the same reasons, although management strategies mostly focus on palliation, we report low rates of post-treatment clinical $(32 \%)$ and radiological $(28.3 \%)$ improvement in patients with spine LMs, comparable to the ones reported in recent studies on spine LMs from primary gliomas (8). In view of the increasing incidence and impact of spine LMs in the current clinical practice, coupled with the growing number of promising new anti-tumoral therapies, physicians and scientists should steer their vision towards tailored treatment approaches targeting molecular and immunologic tumors' microenvironments, simultaneously controlling primary neoplasms, circulating tumor cells, and LMs.

\section{Limitations}

Our study has several limitations. All included articles comprised case reports and retrospective case series subjected to publication and selection biases, and also covered a 42-year time-period characterized by important advances in imaging and treatment protocols, which may have introduced some confounding variables into our analysis. The low level of evidence of included studies precluded a meta-analysis and challenged the statistical power of this study. Finally, pre- and post-treatment patients' performance status scores could not be analyzed and compared due to the lack of granular data.

\section{Conclusion}

Spine LMs represent severe and late complications of systemic malignancies, significantly worsening the functional status of affected patients. In this systematic review of 72 cases, we comprehensively summarized clinical presentation of patients with spine LMs from non-CNS tumors, evaluating the role of available treatments on patients' quality-of-life and survival. Although locoregional radiotherapy, chemotherapy, and surgical decompression are frequently performed for palliation, clinical outcomes are discouraging, and prognoses are dismal. Future studies are required to assess the role of newer molecular and immune treatments in the multimodal management of spine LMs.

\section{Supplementary Material}

Available at: https://www.dropbox.com/sh/u8jaaoh5tewhpqy/AA AWXK1Hx4ZtEixkBez2lIpaa?dl=0

\section{Conflicts of Interest}

The Authors have no relevant financial or non-financial interests to disclose in relation to this study.

\section{Authors' Contributions}

Paolo Palmisciano: Conceptualization, Methodology, Data analysis, Writing - Original draft preparation; Navraj S. Sagoo: Resources, Writing - Reviewing and Editing; Abdurrahman F. Kharbat: Resources, Writing - Reviewing and Editing; Yves J. Kenfack: Resources, Writing - Reviewing and Editing; Gianluca Scalia: Resources, Writing Reviewing and Editing; Giuseppe E. Umana: Resources, Writing Reviewing and Editing; Salah G. Aoun: Resources, Writing Reviewing and Editing; Ali S. Haider: Methodology, Resources, Writing - Reviewing and Editing, Supervision.

\section{References}

1 Le Rhun E and Galanis E: Leptomeningeal metastases of solid cancer. Curr Opin Neurol 29(6): 797-805, 2016. PMID: 27661208. DOI: 10.1097/WCO.0000000000000393

2 Mack F, Baumert BG, Schäfer N, Hattingen E, Scheffler B, Herrlinger U and Glas M: Therapy of leptomeningeal metastasis in solid tumors. Cancer Treat Rev 43: 83-91, 2016. PMID: 26827696. DOI: 10.1016/j.ctrv.2015.12.004

3 Wang N, Bertalan MS and Brastianos PK: Leptomeningeal metastasis from systemic cancer: Review and update on management. Cancer 124(1): 21-35, 2018. PMID: 29165794. DOI: $10.1002 / \mathrm{cncr} .30911$

4 Bhambhvani HP, Rodrigues AJ, Umeh-Garcia MC and Hayden Gephart M: Leptomeningeal carcinomatosis: Molecular 
landscape, current management, and emerging therapies Neurosurg Clin N Am 31(4): 613-625, 2020. PMID: 32921356. DOI: $10.1016 /$ j.nec .2020 .06 .010

5 Scott BJ, Oberheim-Bush NA and Kesari S: Leptomeningeal metastasis in breast cancer - a systematic review. Oncotarget 7(4): 3740-3747, 2016. PMID: 26543235. DOI: 10.18632/ oncotarget.5911

6 Nayar G, Ejikeme T, Chongsathidkiet P, Elsamadicy AA, Blackwell KL, Clarke JM, Lad SP and Fecci PE: Leptomeningeal disease: current diagnostic and therapeutic strategies. Oncotarget 8(42): 73312-73328, 2017. PMID: 29069871. DOI: $10.18632 /$ oncotarget 20272

7 Lawton CD, Nagasawa DT, Yang I, Fessler RG and Smith ZA: Leptomeningeal spinal metastases from glioblastoma multiforme: treatment and management of an uncommon manifestation of disease. J Neurosurg Spine 17(5): 438-448, 2012. PMID: 22958073. DOI: $10.3171 / 2012.7$.SPINE12212

8 Wright $\mathrm{CH}$, Wright J, Onyewadume L, Raghavan A, Lapite I, Casco-Zuleta A, Lagman C, Sajatovic M and Hodges TR: Diagnosis, treatment, and survival in spinal dissemination of primary intracranial glioblastoma: systematic literature review. J Neurosurg Spine: 1-10, 2019. PMID: 31374545. DOI: 10.3171/2019.5.SPINE19164

9 Passarin MG, Sava T, Furlanetto J, Molino A, Nortilli R, Musso AM, Zaninelli M, Franceschi T, Orrico D, Marangoni S, Dealis C, Graiff C, Filippo R, Grisanti S, Simoncini E, Vassalli L, Berruti A and Pedersini R: Leptomeningeal metastasis from solid tumors: a diagnostic and therapeutic challenge. Neurol Sci 36(1): 117-123, 2015. PMID: 25022241. DOI: 10.1007/s10072-0141881-7

10 Lasocki A and Caspersz LJ: T2-SPACE imaging of the cauda equina for the assessment of leptomeningeal metastatic disease. J Clin Neurosci 81: 290-294, 2020. PMID: 33222932. DOI: 10.1016/j.jocn.2020.10.013

11 Chamberlain MC: Comparative spine imaging in leptomeningeal metastases. J Neurooncol 23(3): 233-238, 1995. PMID: 7673985. DOI: $10.1007 / \mathrm{BF} 01059954$

12 Buszek SM and Chung C: Radiotherapy in leptomeningeal disease: a systematic review of randomized and non-randomized trials. Front Oncol 9: 1224, 2019. PMID: 31803614. DOI: 10.3389 /fonc. 2019.01224

13 Kamoda H, Kinoshita H, Yonemoto T, Iuchi T, Tsukanishi T, Hagiwara Y, Ohtori S, Yamazaki M and Ishii T: Factors linked to prognosis in patients with leptomeningeal metastasis diagnosed by spinal magnetic resonance imaging. Spine Surg Relat Res 4(1): 6468, 2019. PMID: 32039299. DOI: 10.22603/ssrr.2019-0064

14 Page MJ, McKenzie JE, Bossuyt PM, Boutron I, Hoffmann TC, Mulrow CD, Shamseer L, Tetzlaff JM, Akl EA, Brennan SE, Chou R, Glanville J, Grimshaw JM, Hróbjartsson A, Lalu MM, Li T, Loder EW, Mayo-Wilson E, McDonald S, McGuinness LA, Stewart LA, Thomas J, Tricco AC, Welch VA, Whiting P and Moher D: The PRISMA 2020 statement: an updated guideline for reporting systematic reviews. BMJ 372: n71, 2021. PMID: 33782057. DOI: 10.1136/bmj.n71

15 Chamberlain M, Junck L, Brandsma D, Soffietti R, Rudà R, Raizer J, Boogerd W, Taillibert S, Groves MD, Le Rhun E, Walker J, van den Bent M, Wen PY and Jaeckle KA: Leptomeningeal metastases: a RANO proposal for response criteria. Neuro Oncol 19(4): 484-492, 2017. PMID: 28039364. DOI: $10.1093 /$ neuonc/now 183
16 Howick J, Chalmers I, Glasziou P, Greenhalgh T, Heneghan C, Liberati A, Moschetti I, Phillips B and Thornton H: Explanation of the 2011 Oxford Centre for Evidence-Based Medicine (OCEBM) Levels of Evidence (Background Document). Oxford Cent Evidence-Based Med, 2011. Available at: https:// www.cebm.ox.ac.uk/resources/levels-of-evidence/ocebm-levelsof-evidence [Last accessed on October 10, 2021]

17 Joanna Briggs Institute: Checklist for Case Reports, 2020. Available at: https://jbi.global/sites/default/files/2021-10/Checklist_for_Case_ Reports.docx [Last accessed on November 15, 2021]

18 Joanna Briggs Institute: Checklist for Case Series, 2020. Available at: https://jbi.global/sites/default/files/2021-10/Checklist_for_Case_ Series.docx [Last accessed on November 15, 2021]

19 Gétaz EP and Miller GJ: Spinal cord involvement in chronic lymphocytic leukemia. Cancer 43(5): 1858-1861, 1979. PMID: 445372. DOI: 10.1002/1097-0142(197905)43:5<1858::aidcncr2820430539>3.0.co;2-e

20 Lange CP, Brouwer RE, Brooimans R and Vecht ChJ: Leptomeningeal disease in chronic lymphocytic leukemia. Clin Neurol Neurosurg 109(10): 896-901, 2007. PMID: 17850954. DOI: 10.1016/j.clineuro.2007.07.021

21 Alicioglu B and Saynak M: Spinal leptomeningeal metastasis in a patient with squamous cell lung cancer. Rev Port Pneumol 14: 875-879, 2008. DOI: 10.1016/S0873-2159(15)30293-2

22 Baek WS and Kubba SV: Cauda equina syndrome due to leptomeningeal carcinomatosis of the ovary. Gynecol Oncol 111(3): 544-545, 2008. PMID: 18433846. DOI: 10.1016/ j.ygyno.2008.03.002

23 Löhr M, Tzouras G, Kocher M, Stenzel W, Reithmeier T, Klug $\mathrm{N}$ and Hampl JA: Treatment strategies of space-occupying intradural metastases of the cauda equina of nonneurogenic origin. Acta Neurochir (Wien) 151(3): 207-215, 2009. PMID: 19247571. DOI: 10.1007/s00701-009-0214-8

24 Mitera G, Roitman D, Pohani G, Brennan M, Fox A, Danjoux C, Sinclair E and Chow E: Leptomeningeal carcinomatosis secondary to gastroesophageal adenocarcinoma: a case report and literature review of a rare occurrence. Scholarly Research Exchange 2009: 1-6, 2011. DOI: 10.3814/2009/642154

25 Dalhaug A, Haukland E and Nieder C: Leptomeningeal carcinomatosis from renal cell cancer: treatment attempt with radiation and sunitinib (case report). World J Surg Oncol 8: 36, 2010. PMID: 20441600. DOI: 10.1186/1477-7819-8-36

26 Hrabalek L, Kalita O, Studentova H, Jankova J, Ehrmann J, Trojanec R and Hajduch M: Intramedullary spinal cord and cauda equina metastasis of breast carcinoma: case report. Biomed Pap Med Fac Univ Palacky Olomouc Czech Repub 154(2): 175-177, 2010. PMID: 20668501. DOI: 10.5507/bp.2010.027

27 Emoto S, Ishigami H, Yamaguchi H, Yamashita H, Kaisaki S and Kitayama J: Frequent development of leptomeningeal carcinomatosis in patients with peritoneal dissemination of gastric cancer. Gastric Cancer 14(4): 390-395, 2011. PMID: 21667135. DOI: 10.1007/s10120-011-0064-y

28 Yildirim R, Gundogdu M, Erdem F, Kiki I and Gundogdu B: Hodgkin's lymphoma with leptomeningeal involvement. Turkish J Hematol 29(3): 283-286, 2012. DOI: 10.5152/tjh.2011.17

29 Cheng K, Chen Y, Zhao F, Gao F, Men HT, Qiu M, Li Q, Bi F and Liu JY: Collecting duct carcinoma presenting uncommon metastatic features. Tumori 98(5): 135e-138e, 2012. PMID: 23235769. DOI: $10.1700 / 1190.13215$ 
$30 \mathrm{Kim}$ KS, Ho SU, Weinberg PE and Lee C: Spinal leptomeningeal infiltration by systemic cancer: myelographic features. AJR Am J Roentgenol 139(2): 361-365, 1982. PMID: 6979894. DOI: 10.2214/ajr.139.2.361

31 Mahta A, Kim RY, Fanta PT, Lawson JD and Kesari S: Leptomeningeal metastasis from appendiceal adenocarcinoma: case report and literature review. J Gastrointest Cancer 43 Suppl 1: 108-110, 2012. PMID: 21826409. DOI: 10.1007/s12029-0119312-y

32 Ren S, Huang Y, Shah P and Manucha V: Metastatic squamous cell carcinoma in cerebrospinal fluid: why a rare diagnosis on cytology? Acta Cytol 56(2): 209-213, 2012. PMID: 22378087. DOI: $10.1159 / 000332377$

33 Wilson B, Sapp C, Abdeen G, Kamona A and Massarweh S: Resolution of extensive leptomeningeal metastasis and clinical spinal cord compression from breast cancer using weekly docetaxel chemotherapy. Breast Cancer Res Treat 131(1): 343346, 2012. PMID: 22037782. DOI: 10.1007/s10549-011-1825-3

34 Zhang M, Mahta A, Kim RY, Akar S and Kesari S: Durable remission of leptomeningeal metastases from hormoneresponsive prostate cancer. Med Oncol 29(2): 806-808, 2012. PMID: 21424585. DOI: 10.1007/s12032-011-9906-Z

35 Gainor JF, Ou SH, Logan J, Borges LF and Shaw AT: The central nervous system as a sanctuary site in ALK-positive nonsmall-cell lung cancer. J Thorac Oncol 8(12): 1570-1573, 2013. PMID: 24389440. DOI: 10.1097/JTO.0000000000000029

36 Kuribayashi H, Abe S, Kuse N, Kusunoki Y, Narato R, Saito H and Gemma A: Marked improvement in leptomeningeal carcinomatosis and spinal cord metastases following alectinib treatment of crizotinib-resistant, ALK-positive lung adenocarcinoma. Int Cancer Conf J 5(2): 69-72, 2015. PMID: 31149429. DOI: $10.1007 / \mathrm{s} 13691-015-0231-9$

37 Lukas RV, Mata-Machado NA, Nicholas MK, Salgia R, Antic T and Villaflor VM: Leptomeningeal carcinomatosis in esophageal cancer: a case series and systematic review of the literature. Dis Esophagus 28(8): 772-781, 2015. PMID: 25142531. DOI: $10.1111 /$ dote 12276

38 Aiyer R, Engelman E, Xue W and Yu E: Dysphagia and anorexia as presentations of leptomeningeal carcinomatosis. BMJ Case Rep 2016: bcr2016214666, 2016. PMID: 27073151. DOI: 10.1136/bcr-2016-214666

39 Alkhotani A, Alrishi N, Alhalabi MS and Hamid T: Cauda equina syndrome secondary to leptomeningeal carcinomatosis of gastroesophageal junction cancer. Case Rep Neurol 8(1): 87-91, 2016. PMID: 27239185. DOI: $10.1159 / 000445869$

40 Bemanian S, Aluru V, Gohari P and Ross M: Poster 21 leptomeningeal carcinomatosis presenting as tetraparesis: a very rare case of small cell prostate carcinoma. PM R $8(9 S)$ : S167, 2016. PMID: 27672788. DOI: 10.1016/j.pmrj. 2016.07.064

41 Barloon TJ, Yuh WT, Yang CJ and Schultz DH: Spinal subarachnoid tumor seeding from intracranial metastasis: MR findings. J Comput Assist Tomogr 11(2): 242-244, 1987. PMID: 3819121. DOI: $10.1097 / 00004728-198703000-00008$

42 Solnes LB, Javadi MS and Rowe SP: 18F-FDG PET/CT imaging of cauda equina syndrome secondary to leptomeningeal metastatic breast cancer. Clin Nucl Med 41(11): e485-e486, 2016. PMID: 27607169. DOI: 10.1097/RLU.0000000000001354

43 Trinh VT, Medina-Flores R and Chohan MO: Leptomeningeal carcinomatosis as primary manifestation of pancreatic cancer. $\mathrm{J}$
Clin Neurosci 30: 124-127, 2016. PMID: 26972704. DOI: 10.1016/j.jocn.2015.12.027

$44 \mathrm{Lu}$ YF, Fong VH, Wu WY, Wang LY and Hsieh $\mathrm{CH}$ : Leptomeningeal metastasis of poorly differentiated uterine cervical adenosquamous carcinoma following reirradiation to metastatic vertebrae: A case report. Medicine (Baltimore) 96(19): e6894, 2017. PMID: 28489796. DOI: 10.1097/MD.0000000000006894

45 Patel BA, Chakor RT, Nadaf S and Kothari KV: Leptomeningeal carcinomatosis presenting as cauda equina syndrome. Ann Indian Acad Neurol 20(3): 320-321, 2017. PMID: 28904471. DOI: 10.4103/aian.AIAN_127_17

46 Yang G, Pan Z, Ma N, Qu L, Yuan T, Pang X, Yang X, Dong L and Liu S: Leptomeningeal metastasis of pulmonary large-cell neuroendocrine carcinoma: A case report and review of the literature. Oncol Lett 14(4): 4282-4286, 2017. PMID: 28943940. DOI: $10.3892 / \mathrm{ol} .2017 .6676$

47 Liu Y, Wang B, Qian Y, Di D, Wang M and Zhang X: Cauda equine syndrome as the primary symptom of leptomeningeal metastases from lung cancer: a case report and review of literature. Onco Targets Ther 11: 5009-5013, 2018. PMID: 30174438. DOI: 10.2147/OTT.S165299

48 Matos F and Cerqueira L: Dural and leptomeningeal spine metastases of breast cancer. Case Reports in Radiology 2019: 14, 2020. DOI: $10.1155 / 2019 / 4289362$

49 Versluis JM, Brandsma D, van den Berg JG and Tesselaar M: Leptomeningeal metastases of a well-differentiated neuroendocrine tumour: a rare entity. BMJ Case Rep 2018: bcr2018226557, 2018. PMID: 30391925. DOI: 10.1136/bcr-2018-226557

50 Zhou ZN, Canon C, Matrai C and Chapman-Davis E: Cauda equina syndrome secondary to leptomeningeal metastases from recurrent primary peritoneal carcinoma. Ecancermedicalscience 12: 814, 2018. PMID: 29515655. DOI: 10.3332/ecancer.2018.814

51 Bernstock JD, Ostby S, Fox B, Sotoudeh H, Janssen A, Kang YJ, Chen J, Prattipati V, Elsayed G, Chagoya G, Yamashita D, Friedman GK, Nabors B, Huh WK and Lobbous M: Cauda equina syndrome in an ovarian malignant-mixed müllerian tumor with leptomeningeal spread. Clin Case Rep 7(12): 2341-2345, 2019. PMID: 31893054. DOI: $10.1002 / \mathrm{ccr} 3.2472$

52 Reggars JW and French SD: A case of leptomeningeal disease presenting as a lumbar nerve root radiculopathy: a case report. Australas Chiropr Osteopathy 7(3): 112-115, 1998. PMID: 17987161.

53 Dagan R, Bryant CM, Mendenhall WM, Amdur RJ, Morris CG, Lanza DC, Dziegielewski PT, Justice JM, Lobo BC, Silver NL, Fernandes R, Bunnell A, Guthrie T, Gopalan PK, Rahman M and Tavanaiepour D: Isolated leptomeningeal progression from sinonasal carcinomas: Implications for staging workup and treatment. Head Neck 41(8): 2647-2654, 2019. PMID: 30908735. DOI: $10.1002 /$ hed.25741

54 Izadi Firouzabadi L, Mead P, Berry J and Hanif S: Cauda equina syndrome due to leptomeningeal carcinomatosis: a medical dilemma. BMJ Case Rep 13(2): e232297, 2020. PMID: 32019757. DOI: 10.1136/bcr-2019-232297

55 Hanout M, Kamil ZS, Alrjoub M, Laperriere N, Altomare F and Krema H: Cauda equina syndrome as the initial presentation of uveal melanoma metastasis. Eur J Ophthalmol: 1120672120944799, 2020. PMID: 32700563. DOI: 10.1177/1120672120944799

56 Nicoletti GF, Umana GE, Graziano F, Calì A, Fricia M, Cicero $\mathrm{S}$ and Scalia G: Cauda equina syndrome caused by lumbar leptomeningeal metastases from lung adenocarcinoma 
mimicking a schwannoma. Surg Neurol Int 11: 225, 2020. PMID: 32874728. DOI: 10.25259/SNI_365_2020

57 Wu RC, Newman W, Patanowitz L, Branstetter BF, Amankulor $\mathrm{N}$ and Tarhini AA: Long-term control of leptomeningeal disease after radiation therapy and nivolumab in a metastatic melanoma patient. Immunotherapy 12(11): 763-769, 2020. PMID: 32571131. DOI: 10.2217/imt-2019-0004

58 Abrar S, Ali N, Qureshi BM and Tahseen R: Central nervous system metastases: a rare presentation of nasopharyngeal carcinoma. BMJ Case Rep 14(8): e243892, 2021. PMID: 34404660. DOI: $10.1136 /$ bcr-2021-243892

59 Tomioka M, Kawase M, Kato D, Takai M, Iinuma K, Horie K, Nakane K, Suzui N, Miyazaki T and Koie T: Leptomeningeal carcinomatosis in urothelial carcinoma of the urinary bladder: a report of a patient with a fulminant course who died of cancer after definitive therapies. Case Rep Urol 2021: 5543939, 2021. PMID: 34012689. DOI: 10.1155/2021/5543939

60 Elliott MA, Letendre L, Li CY, Hoyer JD and Hammack JE: Chronic lymphocytic leukaemia with symptomatic diffuse central nervous system infiltration responding to therapy with systemic fludarabine. Br J Haematol 104(4): 689-694, 1999. PMID: 10192427. DOI: 10.1046/j.1365-2141.1999.01245.x

61 Denissen NH, van Spronsen DJ, Smilde TJ and De Mulder PH: Leptomeningeal carcinomatosis in relapsed non-seminoma testis: a 1-year complete remission with high-dose chemotherapy. Anticancer Drugs 16(8): 897-899, 2005. PMID: 16096440. DOI: 10.1097/01.cad.0000172836.67766.23

62 Deinsberger R, Regatschnig R, Kaiser B and Bankl HC: Spinal leptomeningeal metastases from prostate cancer. J Neurooncol 76(3): 293-297, 2006. PMID: 16151594. DOI: 10.1007/s11060005-7284-0

63 Kastritis E, Moulopoulos LA, Politi E, Kostis E, Pissakas G, Dimopoulos MA and Bamias A: Intramedullary spinal cord and leptomeningeal metastases in a patient with carcinoma of the uterine cervix. Gynecol Oncol 102(1): 124-127, 2006. PMID: 16494929. DOI: 10.1016/j.ygyno.2006.01.025

64 Sullivan LM and Smee R: Leptomeningeal carcinomatosis from perineural invasion of a lip squamous cell carcinoma. Australas Radiol 50(3): 262-266, 2006. PMID: 16732828. DOI: 10.1111/j.1440-1673.2006.01577.x

65 Taillibert S and Chamberlain MC: Leptomeningeal metastasis. Handb Clin Neurol 149: 169-204, 2018. PMID: 29307353. DOI: 10.1016/B978-0-12-811161-1.00013-X

66 Boyle R, Thomas M and Adams JH: Diffuse involvement of the leptomeninges by tumour - a clinical and pathological study of 63 cases. Postgrad Med J 56(653): 149-158, 1980. PMID: 7393804. DOI: $10.1136 /$ pgmj.56.653.149

67 Chamberlain MC: Carcinomatous meningitis. Arch Neurol 54(1): 16-17, 1997. PMID: 9006407. DOI: 10.1001/archneur. 1997.00550130008003

68 Nguyen TK, Sahgal A, Detsky J, Atenafu EG, Myrehaug S, Tseng CL, Husain Z, Heyn C, Maralani P, Ruschin M, Perry J and Soliman H: Predictors of leptomeningeal disease following hypofractionated stereotactic radiotherapy for intact and resected brain metastases. Neuro Oncol 22(1): 84-93, 2020. PMID: 31412120. DOI: $10.1093 /$ neuonc/noz144
69 Palmisciano P, Haider AS, Nwagwu CD, Wahood W, Yu K, Ene CI, O'Brien BJ, Aoun SG, Cohen-Gadol AA and El Ahmadieh TY: The role of immune checkpoint inhibitors in leptomeningeal disease: a systematic review. Anticancer Res 41(11): 5333-5342, 2021. PMID: 34732403. DOI: 10.21873/anticanres.15346

70 Chamberlain MC: Leptomeningeal metastasis. Curr Opin Oncol 22(6): 627-635, 2010. PMID: 20689429. DOI: 10.1097/CCO. 0b013e32833de986

71 Umana GE, Scalia G, Palmisciano P, Passanisi M, Pompili G, Amico P, Ippolito M, Sabini MG, Cicero $S$ and Perrotta R: Spontaneous sacral fracture with associated acrometastasis of the hand. Surg Neurol Int 12: 534, 2021. PMID: 34754584. DOI: 10.25259/SNI_917_2021

72 Passanisi M, Scalia G, Palmisciano P, Franceschini D, Crea A, Capone C, Tranchina MG, Nicoletti GF, Cicero S and Umana GE: Difficulty differentiating between a posterior extradural lumbar tumor versus sequestered disc even with gadolinumenhanced MRI. Surg Neurol Int 12: 267, 2021. PMID: 34221598. DOI: 10.25259/SNI_504_2021

73 Glass JP, Melamed M, Chernik NL and Posner JB: Malignant cells in cerebrospinal fluid (CSF): the meaning of a positive CSF cytology. Neurology 29(10): 1369-1375, 1979. PMID: 573381. DOI: $10.1212 / w n l .29 .10 .1369$

74 Gill CM and Brastianos PK: Management of leptomeningeal carcinomatosis and challenges of trial design. Curr Opin Oncol 31(6): 508-513, 2019. PMID: 31503030. DOI: 10.1097/CCO. 0000000000000574

75 Le Rhun E, Preusser M, van den Bent M, Andratschke N and Weller M: How we treat patients with leptomeningeal metastases. ESMO Open 4(Suppl 2): e000507, 2019. PMID: 31231573. DOI: 10.1136/esmoopen-2019-000507

76 Palmisciano P, Haider AS, Nwagwu CD, Wahood W, Aoun SG, Abdullah KG and El Ahmadieh TY: Bevacizumab vs laser interstitial thermal therapy in cerebral radiation necrosis from brain metastases: a systematic review and meta-analysis. J Neurooncol 154(1): 13-23, 2021. PMID: 34218396. DOI: 10.1007/s 11060-021-03802-x

77 Thomas KH and Ramirez RA: Leptomeningeal disease and the evolving role of molecular targeted therapy and immunotherapy. Ochsner J 17(4): 362-378, 2017. PMID: 29230121. 